RESEARCH PAPER

\title{
Understanding changes in behavior during the COVID-19 pandemic: Opportunities to design around new eating experiences
}

\author{
Mario O. Parra, Luis A. Castro, Jesús Favela
}

Published: 30 November 2021

\begin{abstract}
The pandemic has disrupted many of our daily habits and behaviors. Eating behaviors have been modified by lockdowns due to limited food availability. We report on a qualitative study conducted to better understand these changes in eating behaviors as they represent an opportunity to design technologies that both support healthy eating habits and take advantage of the new conditions produced by the COVID-19 pandemic. We interviewed 14 young professionals in Mexico to understand some of the challenges they faced during the pandemic and how they affected their eating behaviors, including changes in socialization while eating, motivation to improve health, increased cooking, and bingeeating. From this analysis, we envision ways in which novel information technologies can be designed for providing new eating experiences, such as improving virtual socialization while eating, and taking advantage of social media to support cooking and interactions at home.
\end{abstract}

\section{Keywords:}

Eating experiences; COVID-19 Pandemic; Human-centered computing; Human-Computer Interaction; HCI theory; Concepts and models.

\section{Introduction}

The context or circumstances in which individuals consume food shape their eating experience, that is, how one feels while eating and that includes not only the meal, but also the atmosphere [1][2]. For instance, the type of food selected may depend on an emotional state [3], or on the social context, such as eating alone versus eating with family or colleagues [4].

In recent decades, the hospitality industry has helped develop the notion of the meal experience, shaped by issues such as the setting, customer service, and the food itself. Additionally, there has been an increasing interest in eating behaviors within the $\mathrm{HCI}$

Parra, Mario O., Favela, Jesús.

CICESE

Ensenada, México.

marioparra@cicese.edu.mx, favela@cicese.mx

Castro, Luis A.

Instituto Tecnológico de Sonora (ITSON)

Cd. Obregón, México

luis.castro@acm.org community. Late efforts are being directed towards improving wellbeing, creating healthy habits around food, and creating new experiences around food and food design itself [5].

The COVID-19 pandemic [6] has affected practically all aspects of our life, and eating is no exception [7]. Health concerns have motivated some to eat more healthy while catalyzing eating disorders in others [8]. Also, lockdowns of different intensities and durations have closed restaurants and restricted the possibility of eating with others, and food distribution has also been affected.

In this work, we aim at understanding how lockdowns or selfconfinement are shifting the eating experience for young professionals in Mexico. We are interested in young professionals since they are typically independent and flexible with their food choices, and prompt to socialize while eating and drinking. We conducted two rounds of semi-structured interviews with 14 young professionals covering topics related to eating behaviors, and their use of technology during the pandemic. Our findings aim at informing the design of interactive technologies for enriching young professionals' eating experience.

\section{Eating Experience in $\mathrm{HCI}$}

In the last decades, there has been an increasing interest in eating behaviors within the HCI community. Late efforts are being directed towards improving wellbeing, creating healthy habits around food, and creating new experiences around food and food design itself [5]. Several health-related behaviors can be derived from eating activities such as monitoring eating hours [9][10], identifying and analyzing food [11], adherence to a new diet [12], socialization while eating [13], food journaling [14][15], among others. In fact, a recently coined term Human-Food Interaction (HFI) focuses on creating HCI solutions around food [16][17][18]. One of the areas of interest of HFI is that of the eating experience.

It has been reported that the eating experience can be comprised broadly of three factors: the person and her preferences, the service obtained from the location where one is eating, and the attributes of eating out [1]. In this sense, in many ways the eating experience depends on the context of the person. For instance, the type of food selected may depend on mood [3], people choose different types of food and drinks depending on how they are feeling (e.g., anxious, sad, happy). Additionally, the social context is also an important factor for food selection and experience such as eating alone or with other people, cultural ideals, personal factors and resources [4], and some have diet restrictions that modifies their capability to socialize in restaurants [19]. 


\subsection{Eating Patterns and the pandemic in Mexico}

In 2020, the eating experience was disrupted by the COVID-19 pandemic [7]. Mexico has been one of the most affected countries by the pandemic with measures that differed from other OECD countries. For instance, the lockdown was not strict in Mexico in comparison with other countries. As opposed to other countries where social gatherings at private homes were limited to 5 or 10 persons per gathering, in Mexico this was not strictly enforced, although it was advised against. Also, there were no travel restrictions during this time within the country, but restaurants and typically crowded places such as factories and malls were closed for several weeks. The U.S.-Mexico border has remained closed for land travel for over 8 months. Also, to try to curb social gatherings and infections, some local governments enacted dry law for several weeks in April-May 2020 [20], which further helped shape social behaviors and the perception about the pandemic. On the other hand, while there were initial expectations that the pandemic could last for a few weeks or months, intermittent lockdowns and selfconfinement have been going on for over a year and a half, and this has affected expectations and behaviors throughout this period.

In Mexico, eating habits of young adults often worsen when moving away from the family home and assuming primary responsibility for their diets [21]. Likewise, young adults that tend to consume more commercially prepared meals from restaurants instead of self-cooking their meals, have a poorer diet quality [22]. In a local context, the Mexican adult population tends to incur negative eating behaviors such as a high intake of refined foods, sweets, and animal products [23], which is also linked to the obesity problem in the country [24].

The new conditions caused by the pandemic and preventive action by the Mexican government may impact changes in people's eating behaviors [20].

\section{Methods}

We carried out a qualitative study aimed at understanding how the COVID-19 pandemic is impacting people's eating habits, and lifestyle in general. We planned an initial phase of semi-structured interviews with our informants during fall 2020, approximately 6 months into the pandemic.

After that, because of the drastic changes of the pandemic, we decided to plan for a second round of interviews with half of the informants. The main reason was to verify if eating patterns during the pandemic were the same or were modified during these months due to changes in the pandemic, the waves, the information available about potential vaccines, and the like. The time of the second round was during Spring 2021, approximately 12 months into the pandemic.

\section{Research Questions}

Based on the discussion above, our first research question is: How is the pandemic impacting people's eating experience among young professionals in Mexico?

Abrupt changes in eating patterns, increasing awareness of healthy habits, as well as changes in ways people socialize while eating during and after the pandemic, offers an opportunity to envision technologies that could positively impact eating behaviors. This has led to our second research question: What technology design opportunities can potentially form around young professionals' eating experience during and after the COVID-19 pandemic?

\subsection{Informants}

For this study, we recruited 14 young professionals (5 women) living in Mexico. Our informants are young professionals ranging from 23 to 31 years old (four grad students), with a mean age of 26.6 years old (See Table 1). The eligibility criteria were the following: Young adult (20-39 years old), Professionals (college graduate or professional experience), Tech savvy (uses PC or phone regularly/ knows how to use videocalls, and without a diagnosed eating disorder (i.e., bulimia).

We recruited informants from five different cities in northern Mexico via first author's personal contacts and snowball sampling [26] through email and text messages. All the interviews were conducted through Zoom or Meet. Informants signed an informed consent. None of them were compensated for their participation.

Table 1. Informants' demographics

\begin{tabular}{|l|l|l|l|c|}
\hline ID & Gender & Age & Occupation & $\begin{array}{c}\text { Follow-up } \\
\text { interview }\end{array}$ \\
\hline P1 & F & 27 & Teacher & \\
\hline P2 & F & 25 & Merchant & \\
\hline P3 & M & 28 & Graduate student & $\bullet$ \\
\hline P4 & F & 27 & Graphic Designer & $\bullet$ \\
\hline P5 & M & 25 & Graduate student & \\
\hline P6 & M & 31 & Recruiter & $\bullet$ \\
\hline P7 & M & 23 & Graduate student & $\bullet$ \\
\hline P8 & M & 28 & Graduate student & $\bullet$ \\
\hline P9 & M & 25 & Psychologist & \\
\hline P10 & M & 28 & Nutritionist & \\
\hline P11 & F & 25 & Lawyer & \\
\hline P12 & F & 26 & Teacher & \\
\hline P13 & M & 25 & Engineer & \\
\hline P14 & M & 30 & Hotel Worker & \\
\hline
\end{tabular}

\subsection{Data Collection}

We created a semi-structured interview protocol around three main topics: eating habits, general lifestyle, and use of technology. For each topic, we included open ended questions so informants could elaborate and describe their experience with more details. We next include some of the questions: How was your diet before the pandemic started? What other activities do you usually do when you are eating? How do you think technology (or social media) impact the way you eat?

On average, each interview lasted 36 minutes $(\mathrm{SD}=11.5$ minutes). We collected a total of $\approx 12$ hours of interview recordings. Interviews were verbatim transcribed for analysis. Interviews were conducted in Spanish. Quotes translation was overseen by all authors to maintain the intended meaning.

\subsection{Data Analysis}

For analysis, the first author coded interview transcripts line by line generating 174 different single codes. These codes were close to the data. We next present a few examples of the codes: a) feeling stressed because of the pandemic; b) worrying about family 
members; c) follows social media influencers, d) no time for cooking, and e) used to eat out before the pandemic.

The remaining authors checked and reviewed the codes, changing and disregarding codes that were not related to our research questions. Then, in an iterative process similar to Thematic Analysis [27], we reviewed and grouped these codes into four (4) major themes, detailed in the next session.

\section{Results}

From our analysis, four (4) major themes were recurrent in our informants' interviews: 1) negative feelings impacting eating patterns; 2) Finding motivation to improve health; 3) Increased home cooking and eating; and 4) Reduced food socialization. We next explain each of them.

\subsection{Negative feelings impacting eating patterns}

Because of the pandemic, some informants commented how they have more spare time, and this produced some changes in their eating patterns. For instance, P1 stated how this affected her "...normally when I get a lot of spare time... like, you get that kind of anxiety, like I am very idle. What do I do? [well] Eating [I guess], I mean, many people do that". Here, P1 is explaining how more free time creates more opportunities for her to eat, mainly due to feeling anxious, which has been reported as one of the main emotions that trigger eating [28]. For P1, it seemed that sudden changes in her everyday life resulted in having more free time due to perhaps less time spent on traffic or work duties. With outdoor activities severely restricted or unavailable, $\mathrm{P} 1$ could not adjust her activities accordingly and derived feelings of anxiety, which in turn caused her to eat more.

Also, according to our informants, the pandemic caused episodes of binge-eating in some of them. This happens when the person is eating and being distracted, without thinking too much about her food. P1 mentioned how she usually eats when she has some free time, which could be categorized as binge-eating since she is doing it irregularly and without the need to eat.

Binge-eating is a negative eating behavior [29], and according to some of the answers of our informants, the pandemic is causing a negative emotional state that is fomenting this behavior. Some of our informants clearly stated that this behavior was common, although the extent to which this affects people's lives, and why it affected some of our informants but not others, remains an open question.

In this regard, P6 described another scenario "... what my mind was telling me was, stay all day lying on bed, stay asleep, watch TV all day from dawn until you fall asleep, and only get up to eat and go back to bed again.". Here, P6 is describing his days when the pandemic started and how he was feeling apathetic. Since P6 was a recruiter, recruiting staff during the pandemic was different, he could no longer go out to try to recruit people for his company like before, resulting in behaviors such as the one portrayed here.

\subsection{Finding motivation to improve health}

Our informants indicated that they often searched for health support or advice (nutritional and physical) during the pandemic. To some informants, the pandemic motivated them to search for support from health professionals to improve their nutrition and wellness in general. For instance, P7 mentioned the following:

P7: “...during the pandemic I had so much spare time that I decided to workout at home. Then, I used an app that provided home workouts for each day, and that's what I used, with my own weight... But I have been visiting a nutritionist for a month now and
I have a workout and nutritional plan altogether, and they are directed towards the same goals".

In this quote, $\mathrm{P} 7$ describes how he decided to find professional support to improve his wellbeing. His case was not uncommon, some of our informants reported seeking professional advice regarding personal care in terms of weight, nutrition, or physical activity. This was due perhaps to a combination of more spare time and greater interest in being fit derived from the pandemic. Similarly, during the follow-up interview P4 mentioned how she decided to find professional support as well: "I was noticing that sometimes I wasn't eating in a healthy way, and If I tried it one day, at the end of the day I would not meet my goal or plan. Then, I decided to go with a specialist [in nutrition], someone that could guide me in the process". These are two examples of how the pandemic created opportunities and motivation for informants to find professional support for healthier habits. An important aspect here is that $\mathrm{P} 4$ reflects on how professional advice seemed to be more effective for her than self-guided meal plans.

Furthermore, another type of support came from strong ties, which includes parents, significant others, close friends and family members, or roommates. For example, P6 indicated: "At my wife's request, we started changing our diet and become more active. From lying down simply at home, we instead set a goal to go for a walk every day, at least for one hour, and do cardiovascular workouts at home". This quote shows how significant others can greatly impact lifestyle of a person. Even perhaps more important, this illustrates how strong ties can influence behaviors. The influence of others on one's behaviors is an interesting aspect to analyze. Still, with the evidence we collected it is unclear the extent to which strong ties, or weak ties for that matter, influence our informants' behavior. Moreover, it is also unclear the characteristics of our informants or the types of activities that can be used to try to change other people's behaviors.

Likewise, but influenced by close friends (or roommates), P1 mentioned: "With the friends I live with, we support each other or somehow we provide motivation to one another,... like, if one day one [of us] is not in the mood of [anything]..., we say, 'let's workout' and we sort of support each other". This scenario described by P1 illustrates how the support from the people you live with can motivate a person to maintain a positive habit. What is remarkable here is the enormous positive influence that strong ties can have on the informants' behavior. Beyond inviting people to do positive activities, emotional support seems to be key in tough moments such those experienced by some of our informants during the pandemic.

Generally, family members and close friends have influence in most aspects of the life of a person. This is the reason why some behaviors are formed around the people you spend the most time with i.e., social circles, and eating behavior are not an exception to this rule.

\subsection{Increased home cooking and eating}

Because of the lockdown and self-confinement, our informants were temporarily forced to eat at home all the time for some time. All restaurants, and most shops (except those deemed essential) closed their doors to prevent people from being infected of COVID19. This situation resulted in increased home cooking by participants.

As stated by most of our informants, they cooked their own meals more frequently. For example, P10 explained the following: "Oddly, I noted how I could eat more meals cooked by myself. I think that part is interesting because [before the pandemic] I used to cook, but [only] once for the whole week and with less variety of 
dishes, and when the pandemic started, my variety of food increased". For P10, the pandemic gave him more time for cooking, an activity that incremented his dish variety. Although not mentioned by P10, a nutritionist in his late twenties, this process perhaps comprised reading more about different ingredients, shopping in local markets or online, and several others that are related to diversify the preparation of meals and even ingredients.

Similarly, P12 mentioned how the lockdown gave her more time for cooking as well, but she says her meals were not necessarily nutritious "At noon we [she and her family] would eat as usual, but I now had more time to cook, [so] I did my recipes that were not that healthy at all such as pizza, boneless [spicy chicken] ... everything that I couldn't get from the street anymore, I would cook them for myself'. At least for P12, this craving for street food seemed to be related to nostalgia, and perhaps a way to cope with the pandemic. That is, preparing street food could be a way to relate to activities that were suddenly unavailable (e.g., eating out, having drinks/snacks with friends).

Another interesting activity that we found was the apparent practice of mindful eating by one of our informants. In this practice people focus only on their food and avoid distractions, and has been reported to be effective in addressing binge eating, emotional eating and eating in response to external cues [30]. P2 describes what she does now when eating alone at home "I don't watch much TV, but I try to stay away from the mobile phone when I'm eating. I have read and heard that it is really good to focus just like that on your meal and your eating... but I don't always do it, there are days that I do it and others that I don't. The TV certainly I don 't [watch it]." Although P2 does not do this every day, it seems to be important for her.

Nevertheless, not all informants mentioned anything about these types of practices, others watch TV or use their phone, as mentioned by P11, a female lawyer: "sometimes I just start like watching TV and at the same time I'm eating, but most of the times I'm just at the table, but on my phone or talking with whoever else is also eating." Here P11 does not seem to think the activity of eating is important enough to pay full attention to it, as she prefers to be socializing or distracted while eating, which could be a way of distraction from work duties.

What we can see with these examples is that the context created by the pandemic (more free time, eating at home, and for some without company) could generate more occasions to practice mindful eating for a few people. However, as informed by P11, not everyone has an interest in this form of activity, some rather enjoy this time by doing something else such as watching TV or socializing with others.

\subsection{Reduced food socialization}

The restrictions imposed by the lockdown changed how people socialize, and this has also impacted the lives of the informants. The lockdown imposed by the Mexican government prevented people from going out to dine at restaurants, at least at the beginning of the pandemic. This derived in sudden changes in the lifestyle of the informants. For example, P3 described his new mindset "I think that the new mindset is the vision you have with respect to social activities. Before, it was quite common to say: 'let's go eat, let's go for a pizza, let's go grab a beer,' or something like that, and now we can't. Now [with the pandemic] the consumption of that kind of food has been reduced." As mentioned by P3, they must adapt to the new normal, since social gatherings at restaurants are limited to prevent people from spreading the virus. These and similar restriction in public places in Mexico represented serious challenges for young professionals, particularly those who may be living on their own in a city different from where they grew up. That is, some of our informants did not have access to public places to meet up, and without any private home to visit, this might be emotionally challenging. Still, it is remarkable that $\mathrm{P} 3$ focuses on the reduction of consumption of "that kind of food" i.e., street or junk food, rather than on emotional aspects.

Likewise, a similar situation occurred to P12, here she mentions what she used to do before the pandemic "I would normally eat out in the evenings, like at a movie theater, and then getting back home from the movies, it was like I wouldn't eat anything. Popcorn and soda were my dinner for that day. That was always on Mondays, it was rare for me not to go to the movies on Mondays. Other meals I would eat out were really diverse... I could say every 2 or 3 weeks, and during the weekends, I would go to a restaurant for dinner or a café. I can say that was almost every weekend, and on average 3 times a week I would go eat out and it was normally for dinner." Now, all the activities described by P12 were not viable during the start of the pandemic, and still limited after 10 months of pandemic. What is more interesting of P12's comment is that it somehow implies that several times a week she socialized and mingled with others while doing activities such as grabbing a bite, going to the movies, or going for coffee. In her comment, she seems to reflect on the low nutritional content of the dinner for her typical Monday, but she also seems to proudly contrast her other days where she would obtain a really "diverse" menu.

Because of this, the lockdown and self-confinement are causing critical changes in our informants' lifestyle. Many of the activities they used to do before the pandemic are now unavailable or limited, which is shifting social behaviors related to eating patterns, and they will probably be preserved for some time.

\section{Design Opportunities}

Based on our results, we next discuss some ways in which the design space of interactive technologies to improve eating experiences can be rethought, according to the four main themes discussed in the previous section.

\subsection{Binge Eating}

In relation to the first theme of negative feelings impacting eating patterns, our informants reported that during the pandemic they felt low, apathetic, stressed, and experiencing other negative emotions or states that provoked episodes of binge eating. Therefore, we envision applications capable of monitoring not only eating habits, but also users' emotional state, and offer recommendations according to their mood or to try boosting their mood (i.e., "If you're feeling low and hungry, eating a healthy snack like baby carrots may help you feel better").

Additionally, it is necessary to monitor with accuracy people's eating moments to be able to prevent or detect binge eating episodes. Monitoring eating outside of the lab is still a complex problem. There are many advances in this activity, for instance, some focus on detecting eating episodes through video [31], and others try to count chewing movements with muscle contractions utilizing an accelerometer [32]. Nonetheless, despite of the advances on this area, monitoring eating in the real life is still complex and remains an open problem. For all of this, being able to detect the exact moment a binge eating episode is happening is an opportunity for design.

\subsection{Strong Ties}

For the second theme, we found that our informants are motivated to being and feeling healthier. Our informants showed us how they 
look for support from their strong ties, such as significant others, parents, close friends, or roommates. For this, we envision applications where people can obtain motivation, recommendations, and support from strong ties. There is previous research focused on understanding and experimenting with social media interactions/motivation to improve personal health [33].

Further, in case of a reduced number of strong ties (i.e., some older adults), there is work on using conversation agents for improving eating behaviors and decrease loneliness [34]. We find conversational agents are a great opportunity for people to interact in a natural way, and to have someone (or something) programmed to motivate and support behavior change focused on improving eating patterns. For instance, late works on conversational agents have shown how they are being utilized for behavior change and motivation [35][36].

\subsection{Mindful Eating/Cooking}

The pandemic also caused our informants to cook and eat more often at home, since people were mostly at home and restaurants closed for eating in. If this becomes a habit, this could be beneficial since it favors healthy eating [37]. Therefore, cooking is another opportunity for design and creating new ways to socialize around food. Online interactions with friends could enhance cooking and make it more amusing for people.

Additionally, one of our informants reported about practices related to mindful eating. This practice predicts healthy behavior for people who use it daily [30]. Although, there is not enough research in the HCI community to create applications or prototypes to enhance this practice in particular, there is related research in the general practice of mindfulness [38]. We consider this activity of mindful eating a viable option for the creation of new technology. Previous research has explored this topic, such as a smart spoon project [39], but this is still a relatively unexplored area.

\subsection{Social Eating}

Lastly, a reduction in food socialization was commonplace across most informants. Lockdown-driven restrictions seem to have changed the way people socialize while eating. Thus, designs should consider other ways of socialization supported by information technology. There have been many efforts on finding new ways to socialize while eating [40][41][42][43]. Some motivate connecting with strangers [44], others focus on interactions on social media [45][33], and others utilize gamification [46]. However, the designs of the new technologies should focus not only on replacing face to face with virtual interactions, but also should consider what aspect of the eating experience interactive technologies can create, shift, or augment (e.g., food, taste, music, mingling).

In this matter, we envision ways for technology to design new environments for interaction while eating. During the pandemic, new applications for this kind of interactions started rising. For instance, a new platform called Gather Town (gather.town) allows users to create virtual spaces in which people can interact with virtual objects moving their avatar across the space. It also allows users to interact with other people simulating videocalls (with cameras and microphones) when you are close to other avatars in the virtual space. These types of virtual interactions generate new opportunities for socializing while eating, for example, a virtual restaurant could be designed where people simulate their social activities before the pandemic.

\section{Conclusions}

The eating experience of young professionals in Mexico, as for most people worldwide, has been heavily impacted by the COVID19 pandemic. The results from the qualitative study described in this article, derived in four main themes in which the eating experience changed. In general, eating patterns have been modified by negative feelings, people are now looking for support to improve health, people are cooking and eating mostly from home, and food socialization has decreased and drastically changed. Some of these changes may stay for a while, whereas others may be driven merely by government rules.

In any case, this long-term experience can motivate the adoption of habits and technologies that could positively impact the eating experience. For this, we also described four main opportunities for design: binge-eating, strong ties, mindful eating/cooking, and social eating. Future work includes co-design sessions with young professionals to unravel more specific forms in which technology can enhance their eating experience.

\section{Acknowledgments}

The research was partially funded by CONACYT grant number A1-S-11287 and through a graduate scholarship provided to the first author.

\section{References}

[1] N. Johns, P. Tyas, T. Ingold, and S. Hopkinson, "Investigation of the perceived components of the meal experience, using perceptual gap methodology," Prog. Tour. Hosp. Res., vol. 2, no. 1, pp. 15-26, Mar. 1996.

[2] R. Leung and P. T. Loo, "Co-creating interactive dining experiences via interconnected and interoperable smart technology," Asian J. Technol. Innov., 2020, doi: 10.1080/19761597.2020.1822748.

[3] J. R. E. Fox and K. Froom, "Eating disorders: A basic emotion perspective," Clin. Psychol. Psychother., vol. 16, no. 4, pp. 328-335, Jul. 2009, doi: 10.1002/cpp.622.

[4] J. Sobal and C. A. Bisogni, "Constructing Food Choice Decisions," Ann. Behav. Med., vol. 38, no. S1, pp. 37-46, Dec. 2009, doi: 10.1007/s12160-009-9124-5.

[5] J. Deng et al., "The Future of Human-Food Interaction," in Conference on Human Factors in Computing Systems Proceedings, May 2021, pp. 1-6, doi: 10.1145/3411763.3441312.

[6] M. Ciotti, M. Ciccozzi, A. Terrinoni, W.-C. Jiang, C.-B. Wang, and S. Bernardini, "The COVID-19 pandemic," Crit. Rev. Clin. Lab. Sci., vol. 57, no. 6, pp. 365-388, Aug. 2020, doi: 10.1080/10408363.2020.1783198.

[7] V. Flaudias et al., "COVID-19 pandemic lockdown and problematic eating behaviors in a student population," $J$. Behav. Addict., vol. 9, no. 3, pp. 826-835, Sep. 2020, doi: 10.1556/2006.2020.00053.

[8] L. Di Renzo et al., "Eating habits and lifestyle changes during COVID-19 lockdown: An Italian survey," J. Transl. Med., vol. 18, no. 1, p. 229, Jun. 2020, doi: 10.1186/s12967020-02399-5.

[9] T. Vu, F. Lin, N. Alshurafa, and W. Xu, "Wearable Food Intake Monitoring Technologies: A Comprehensive Review," Computers, vol. 6, no. 1, p. 4, Jan. 2017, doi: $10.3390 /$ computers6010004. 
[10] J. Blair, Y. Luo, N. F. Ma, S. Lee, and E. K. Choe, "OneNote Meal: A Photo-Based Diary Study for Reflective Meal Tracking," AMIA ... Annu. Symp. proceedings. AMIA Symp., vol. 2018, pp. 252-261, 2018, Accessed: Aug. 11, 2021. [Online]. Available: /pmc/articles/PMC6371351/.

[11] C. J. Boushey, M. Spoden, F. M. Zhu, E. J. Delp, and D. A. Kerr, "New mobile methods for dietary assessment: Review of image-assisted and image-based dietary assessment methods," in Proceedings of the Nutrition Society, Aug. 2017, vol. 76, no. 3, pp. 283-294, doi: 10.1017/S0029665116002913.

[12] Q. Wang, B. Egelandsdal, G. V. Amdam, V. L. Almli, and M. Oostindjer, "Diet and physical activity apps: Perceived effectiveness by app users," JMIR mHealth uHealth, vol. 4, no. 2, p. e5114, Jun. 2016, doi: 10.2196/mhealth.5114.

[13] C. Spence, M. Mancini, and G. Huisman, "Digital Commensality: Eating and Drinking in the Company of Technology," Frontiers in Psychology, vol. 10. Frontiers Media S.A., p. 2252, Oct. 09, 2019, doi: 10.3389/fpsyg.2019.02252.

[14] P. Achananuparp, E. P. Lim, and V. Abhishek, "Does journaling encourage healthier choices? Analyzing healthy eating behaviors of food journalers," in ACM International Conference Proceeding Series, Apr. 2018, vol. 2018-April, pp. 35-44, doi: 10.1145/3194658.3194663.

[15] Y. Luo, Y.-H. Kim, B. Lee, N. Hassan, and E. K. Choe, "FoodScrap: Promoting Rich Data Capture and Reflective Food Journaling Through Speech Input," in Designing Interactive Systems Conference 2021, Jun. 2021, vol. 13, no. 21, pp. 606-618, doi: 10.1145/3461778.3462074.

[16] R. A. Khot and F. Mueller, "Human-food interaction," Found. Trends Human-Computer Interact., vol. 12, no. 4, pp. 238-415, 2019, doi: 10.1561/1100000074.

[17] A. Grimes and R. Harper, "Celebratory technology: New directions for food research in HCI," in Conference on Human Factors in Computing Systems - Proceedings, 2008, pp. 467-476, doi: 10.1145/1357054.1357130.

[18] F. A. Bertran, S. Jhaveri, R. Lutz, K. Isbister, and D. Wilde, "Making sense of human-food interaction," in Conference on Human Factors in Computing Systems - Proceedings, May 2019, pp. 1-13, doi: 10.1145/3290605.3300908.

[19] F. Nunes, J. Almeida, C.-F. Chung, and N. Verdezoto, "Avoiding Reactions Outside the Home: Challenges, Strategies, and Opportunities to Enhance Dining Out Experiences of People with Food Hypersensitivities," in Proceedings of the 2021 CHI Conference on Human Factors in Computing Systems, May 2021, vol. 16, no. 21, pp. 1-16, doi: $10.1145 / 3411764.3445662$.

[20] I. Ibarra-Nava, J. A. Cardenas-De La Garza, R. E. RuizLozano, and R. G. Salazar-Montalvo, "Mexico and the COVID-19 Response," Disaster Med. Public Health Prep., vol. 14, no. 4, pp. E17-E18, Aug. 2020, doi: 10.1017/dmp.2020.260.

[21] A. Papadaki, G. Hondros, J. A. Scott, and M. Kapsokefalou, "Eating habits of University students living at, or away from home in Greece," Appetite, vol. 49, no. 1, pp. 169-176, Jul. 2007, doi: 10.1016/j.appet.2007.01.008.
[22] M. G. Thorpe, M. Kestin, L. J. Riddell, R. S. Keast, and S. A. McNaughton, "Diet quality in young adults and its association with food-related behaviours," Public Health Nutr., vol. 17, no. 8, pp. 1767-1775, Aug. 2014, doi: $10.1017 / \mathrm{S} 1368980013001924$.

[23] M. Flores et al., "Dietary Patterns in Mexican Adults Are Associated with Risk of Being Overweight or Obese," J. Nutr., vol. 140, no. 10, pp. 1869-1873, Oct. 2010, doi: $10.3945 /$ jn. 110.121533 .

[24] J. Dávila-Torres, J. De Jesús González-Izquierdo, and A. Barrera-Cruz, "Medicina social Panorama de la obesidad en México," Apr. 2015.

[25] B. Mendez Bahena, J. C. Moreno, P. Cruz Hernandez, and M. Metropolitan, "Covid-19 crisis management in Mexico: initial reopening / Covid-19 gestão de crise no México: reabertura inicial," Rev. Direito da Cid., vol. 13, no. 2, pp. 541-563, Apr. 2021, doi: 10.12957/rdc.2021.53644.

[26] Leo A. Goodman, "Snowball Sampling on JSTOR,” Publ. By Inst. Math. Stat., pp. 148-170, 1961, Accessed: Aug. 16, 2021. [Online]. Available:

https://www.jstor.org/stable/2237615.

[27] V. Braun and V. Clarke, "Thematic analysis.," in $A P A$ handbook of research methods in psychology, Vol 2: Research designs: Quantitative, qualitative, neuropsychological, and biological., Washington: American Psychological Association, 2012, pp. 57-71.

[28] J. Polivy, C. P. Herman, and T. McFarlane, "Effects of Anxiety on Eating: Does Palatability Moderate DistressInduced Overeating in Dieters?," J. Abnorm. Psychol., vol. 103 , no. 3, pp. 505-510, 1994, doi: 10.1037/0021843X.103.3.505.

[29] T. F. Heatherton and R. F. Baumeister, "Binge eating as escape from self-awareness," Psychol. Bull., vol. 110, no. 1, pp. 86-108, 1991, doi: 10.1037/0033-2909.110.1.86.

[30] J. M. Warren, N. Smith, and M. Ashwell, “A structured literature review on the role of mindfulness, mindful eating and intuitive eating in changing eating behaviours: Effectiveness and associated potential mechanisms," Nutrition Research Reviews, vol. 30, no. 2. Cambridge University Press, pp. 272-283, Dec. 01, 2017, doi: $10.1017 /$ S0954422417000154.

[31] D. Hossain, T. Ghosh, and E. Sazonov, "Automatic Count of Bites and Chews From Videos of Eating Episodes," IEEE Access, vol. 8, pp. 101934-101945, 2020, doi: 10.1109/ACCESS.2020.2998716.

[32] S. Wang et al., "Eating detection and chews counting through sensing mastication muscle contraction," Smart Heal., vol. 9-10, pp. 179-191, Dec. 2018, doi: 10.1016/j.smhl.2018.07.004.

[33] C. F. Chung, E. Agapie, J. Schroeder, S. Mishra, J. Fogarty, and S. A. Munson, "When personal tracking becomes social: Examining the use of instagram for healthy eating," in Conference on Human Factors in Computing Systems Proceedings, May 2017, vol. 2017-May, pp. 1674-1687, doi: $10.1145 / 3025453.3025747$.

[34] L. L. Kramer, B. C. Mulder, L. van Velsen, and E. de Vet, "Use and Effect of Web-Based Embodied Conversational Agents for Improving Eating Behavior and Decreasing 
Loneliness Among Community-Dwelling Older Adults: Protocol for a Randomized Controlled Trial," JMIR Res. Protoc., vol. 10, no. 1, p. e22186, Jan. 2021, doi: $10.2196 / 22186$.

[35] M. El Kamali, L. Angelini, D. Lalanne, O. Abou Khaled, and E. Mugellini, "Multimodal conversational agent for older adults' behavioral change," in ICMI 2020 Companion Companion Publication of the 2020 International Conference on Multimodal Interaction, Oct. 2020, pp. 270 274, doi: 10.1145/3395035.3425315.

[36] V. Carfora, F. Di Massimo, R. Rastelli, P. Catellani, and M. Piastra, "Dialogue management in conversational agents through psychology of persuasion and machine learning," Multimed. Tools Appl., vol. 79, no. 47-48, pp. 35949-35971, Dec. 2020, doi: 10.1007/s11042-020-09178-w.

[37] P. Monsivais, A. Aggarwal, and A. Drewnowski, "Time Spent on Home Food Preparation and Indicators of Healthy Eating," Am. J. Prev. Med., vol. 47, no. 6, pp. 796-802, Dec. 2014, doi: 10.1016/j.amepre.2014.07.033.

[38] Y. Akama and A. Light, "Towards mindfulness: Between a detour and a portal," in Conference on Human Factors in Computing Systems - Proceedings, Apr. 2015, vol. 18, pp. 625-634, doi: 10.1145/2702613.2732498.

[39] J. Y. L. Yi, D. Aggarwal, and R. A. Khot, "SWAN: Designing a companion spoon for mindful eating," in TEI 2020 - Proceedings of the 14th International Conference on Tangible, Embedded, and Embodied Interaction, Feb. 2020, pp. 743-756, doi: 10.1145/3374920.3375009.

[40] J. Sandbulte, C.-H. Tsai, and J. M. Carroll, "Working Together in a PhamilySpace," Proc. ACM Human-Computer Interact., vol. 5, no. CSCW1, pp. 1-32, Apr. 2021, doi: $10.1145 / 3449198$.
[41] A. Panicker, K. Basu, and C.-F. Chung, "Changing Roles and Contexts: Symbolic Interactionism in the Sharing of Food and Eating Practices between Remote, Intergenerational Family Members," Proc. ACM Human-Computer Interact., vol. 4, no. CSCW1, pp. 1-19, May 2020, doi: $10.1145 / 3392848$.

[42] C. Schaefbauer, D. Kahn, A. Le, G. Sczechowski, and K. Siek, "Snack buddy: Supporting healthy snacking in low socioeconomic status families," in CSCW 2015 -

Proceedings of the 2015 ACM International Conference on Computer-Supported Cooperative Work and Social Computing, Feb. 2015, pp. 1045-1057, doi: 10.1145/2675133.2675180.

[43] K. Lukoff, T. Li, Y. Zhuang, and B. Y. Lim, "TableChat: Mobile food journaling to facilitate family support for healthy eating," Proc. ACM Human-Computer Interact., vol. 2, no. CSCW, pp. 1-28, Nov. 2018, doi: 10.1145/3274383.

[44] Y. Wu, E. Krebs, A. Hassan Shankaranand, P. Shih, and C. F. Chung, "Meal chat: Promoting mealtime social interaction for college students," in Conference on Human Factors in Computing Systems - Proceedings, Apr. 2020, pp. 1-8, doi: 10.1145/3334480.3382822.

[45] T. T. Phan and D. Gatica-Perez, "\#Healthy \#Fondue \#Dinner: Analysis and inference of food and drink consumption patterns on Instagram," in ACM International Conference Proceeding Series, Nov. 2017, pp. 327-338, doi: $10.1145 / 3152832.3152857$.

[46] S. Olinsky et al., "Meals for Monsters: A Mobile Application for the Feasibility of Gaming and Social Mechanisms," in Conference on Human Factors in Computing Systems Proceedings, May 2021, pp. 1-9, doi: 10.1145/3411763.3451789.

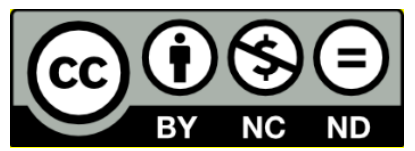

(C) 2021 by the authors. This work is licensed under the Creative Commons AttributionNonCommercial-NoDerivatives 4.0 International License. To view a copy of this license, visit http://creativecommons.org/licenses/by-nc-nd/4.0/ or send a letter to Creative Commons, PO Box 1866, Mountain View, CA 94042, USA. 